EPJ Web of Conferences 41, 10003 (2013)

DOI: $10.1051 /$ epjconf/20134110003

C) Owned by the authors, published by EDP Sciences, 2013

\title{
Mid-Infrared femtosecond filament and three octaves continuum generation in gases
}

\author{
S. Ališauskas, ${ }^{1}$ D. Kartashov, ${ }^{1}$ A. Pugžlis, ${ }^{1}$ A. Voronin, ${ }^{2}$ A. Zheltikov, ${ }^{2,3}$ M. Petrarca, ${ }^{4}$ P. Bejot, ${ }^{4}$ \\ J. Kasparian, ${ }^{4}$ and A. Baltuška ${ }^{1}$ \\ ${ }^{1}$ Photonics Institute Vienna University of Technology, Gusshausstrasse 27-387, A-1040 Vienna, \\ Austria \\ ${ }^{2}$ Physics Department, International Laser Center, M.V. Lomonosov Moscow State University, \\ 119992 Moscow, Russia \\ ${ }^{3}$ Department of Physics and Astronomy, Texas A\&M University, College Station TX, 77843-4242, \\ USA \\ ${ }^{4}$ Université de Genève, Rue de l'École de Médecine 20, 1211 Genève, Switzerland
}

\begin{abstract}
We report experimental and theoretical results on mid-infrared femtosecond pulse filamentation in different gases. Highly efficient generation of a three-octave-wide spectral continuum in argon, covering the main atmospheric transparency windows, is demonstrated and explained.
\end{abstract}

\section{Introduction}

Being a fascinating versatile spatio-temporal nonlinear optical phenomenon, femtosecond filaments serve as prospective light sources in spectroscopy, particularly in standoff atmospheric detection, high-voltage gas discharge triggers, teraherz emission sources, etc. [1]. To date, experimental research on femtosecond pulse filamentation has been almost exclusively carried out at the wavelength of $0.8 \mu \mathrm{m}$. Femtosecond filamentation with long-wavelength sources presents a daunting challenge because of the $P_{\mathrm{cr}} \propto \lambda^{2}$ scaling of the critical power of self-focusing as a function of the laser wavelength. We report on the first, to our knowledge, experimental observation of filamentation in gases of eye-safe femtosecond mid-IR pulses leading to the generation of a supercontinuum across the full breadth of the main spectral transparency windows of the atmosphere.

\section{Experimental results}

Experiments were performed with an OPCPA system delivering 12-mJ, 80-fs ( $>130 \mathrm{GW}$ peak power) pulses centered at $3.9 \mu \mathrm{m}$ at the repetition rate of $20 \mathrm{~Hz}$ [2]. We estimated the critical power for self-focusing by extrapolating the value known at $0.8 \mu \mathrm{m}$ according to the $\lambda^{2}$ scaling law. The estimate of $\sim 200 \mathrm{GW}$ corresponding to atmospheric pressure is beyond the current performance of the laser source and the deficit of peak power was compensated by working with a pressurized gas cell.

This is an Open Access article distributed under the terms of the Creative Commons Attribution License 2.0, which permits unrestricted use, distribution, and reproduction in any medium, provided the original work is properly cited. 

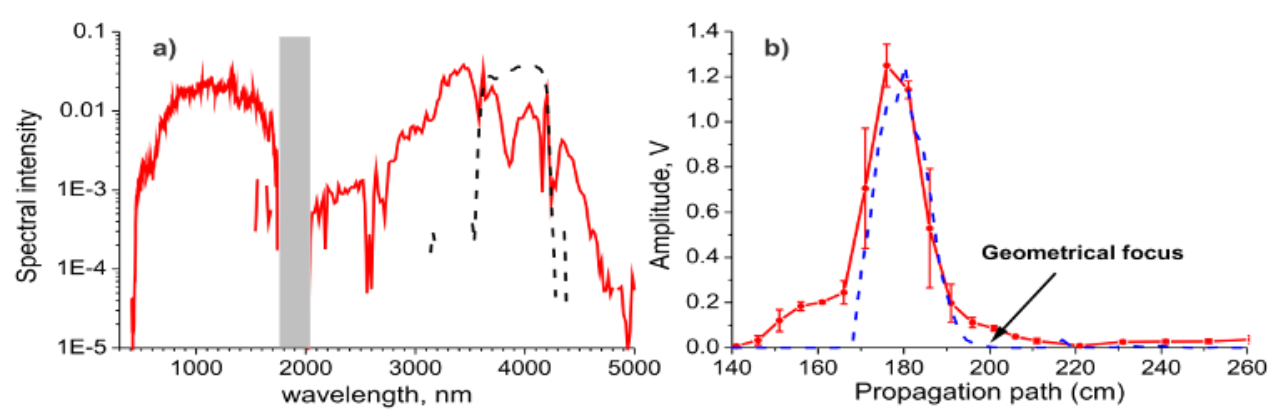

Fig. 1. a) Spectrum after the gas cell filled by 4.5 bar of Ar. Pulse energy is $6.5 \mathrm{~mJ}$. The dotted line represents the input pulse spectrum. The shadowed bar shows the "blind" region not covered by the spectrometers. b) Longitudinal plasma distribution in the mid-IR filament measured by the electrodes.

The beam was softly focused $\left(f=2 \mathrm{~m}\right.$ ) into a $4.7-\mathrm{m}$-long gas cell capped with $3-\mathrm{mm}$ thick $\mathrm{CaF}_{2}$ Brewster windows. The output beam was imaged on a pyroelectric camera. The spectra were recorded piece-wise in the ranges 350-1700 nm (AQ-6315A, Ando) and 2-6 $\mu \mathrm{m}$ with a $1 / 2$-meter scanning monochromator (Digikröm, CVI) and a liquid nitrogen cooled InSb photodetector (J10D, Teledyne Judson). The two spectra were pieced together upon calibrating the energy content in each spectral region using a coarse prism spectrometer and relying on a flat spectral response of a multichannel pyroelectric detector.

Characterization of the longitudinal plasma distribution in the filament was carried out with two movable electrodes inside the cell spaced by $1 \mathrm{~cm}$ and biased at $500 \mathrm{~V}$. The current flowing between the electrodes is proportional to the plasma concentration [3].

Filamentation in argon starts at the pressure $<3$ bar and is manifested by a shift of the focus toward the laser source, appearance of plasma as confirmed by a current between the electrodes, and a significant contraction of the output beam size. Increasing the pressure to 4 bar results in a dazzling white-light beam surrounded by a ring structure of conical emission. The spectrum measured in argon is given in Fig.1a and spans from the UV to above $5 \mu \mathrm{m}$. The filament length measured with the electrodes in this case is about $60 \mathrm{~cm}$ (Fig. 1b). The overall energy efficiency of frequency conversion from the mid-IR fundamental spectrum to the UV-near-IR spectral range from $350 \mathrm{~nm}$ to $1.7 \mu \mathrm{m}$ was measured to be up to $4 \%$.

To gain insight into the mechanism behind such efficient supercontinuum generation, we have investigated the spectral dynamics as a function of argon pressure (Fig.2). In this experiment, the spectrum in the UV-visible spectral range was measured with the HR4000 Ocean Optics

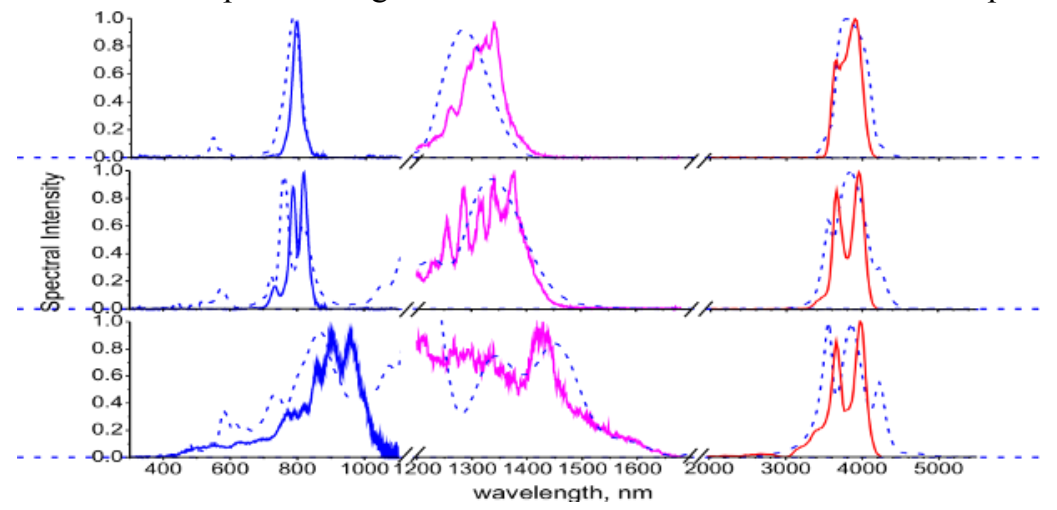

Fig. 2. Spectra of the fifth and third harmonics and of the fundamental for different values of argon pressure. The solid lines represent experimentally measured spectra, the dashed lines represent the results of numerical simulations. Note that each harmonic spectrum is independently normalized to 1 . 


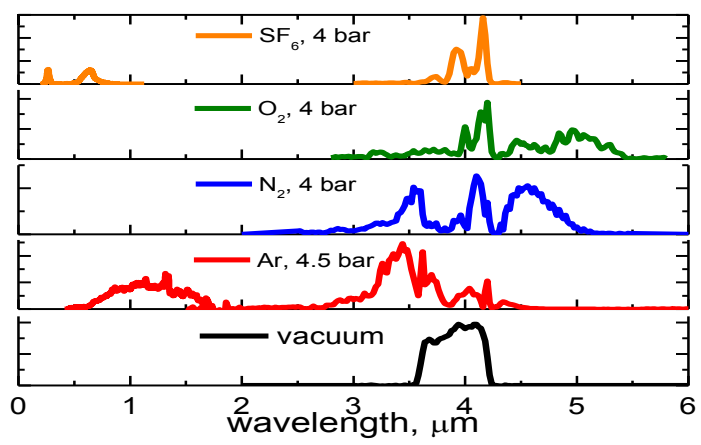

Fig. 3. Spectra of the filament in argon and different molecular gases.

spectrometer. Coupling into the spectrometer was optimized for measurements of the fifth harmonic. The measurements have shown that the supercontinuum builds up via an efficient four-wave mixing between the fundamental $3.9 \mu \mathrm{m}$ light and its harmonics generated in the gas. At lower gas pressures ( $1 \mathrm{bar})$, the spectrum of the third and fifth harmonics corresponds to a frequency-converted image of the spectral broadening at the fundamental wavelength. At higher pressures, generation of the fifth harmonics via a cascaded four-wave mixing between the fundamental and third harmonics pulses becomes prominent. Approaching filamentation threshold around 3.5 bar, the interaction length dramatically increases, which is accompanied by a significant enhancement of spectral broadening at the fundamental and harmonics frequencies. In combination with the plasma blue shift, this spectral broadening leads to the formation of an ultra-broadband spectral continuum.

Our numerical modeling confirms the inferred scenario for the observed highly efficient continuum generation in the mid-IR filament. Filamentation is modeled by numerically solving the $(3+1)$-dimensional nonlinear Schrödinger equation, which accounts for plasma dispersion and refraction, beam diffraction, as well as Kerr, Raman, and plasma nonlinearities. Theoretical results presented as dashed curves in Fig.2 convincingly agree with the experiment. They confirm the crucial importance of the four-wave mixing processes between the fundamental radiation and its harmonics for efficient continuum generation, as it was suggested earlier in [4]. Also, they highlight a significant role of fifth-order nonlinearity in the dynamics of the mid-IR filament.

Alongside the measurements of mid-IR filamentation in argon, we performed experiments with different molecular gases such as nitrogen, oxygen and $\mathrm{SF}_{6}$ both for focused and collimated beams. Here, we succeeded in filamenting collimated input mid-IR beams in a 6-m-long gas cell filled by gases at pressures up to 8 bar. Comparison of filamentation in molecular gases like nitrogen and oxygen with filamentation in argon has shown a drastic difference in the spectral and spatial dynamics of the femtosecond mid-IR laser radiation. As opposed to argon, the filamentation in molecular gases produces a substantially stronger spectral broadening on the mid-IR side and a much weaker broadening in the visible - near-IR range (Fig.3). Intriguingly, we also observed the effect of self-focusing suppression in nitrogen for a high-intensity collimated input beam, which prevents the onset of filamentation. In the case of filamentation in $\mathrm{SF}_{6}$, an unexpectedly strong UV emission in the spectral range 200-400 $\mathrm{nm}$ was observed (Fig.3). Our results demonstrate an incredibly rich variety of new nonlinear optical phenomena accompanying mid-IR filamentation.

\section{References}

1. S.L. Chin "Femtosecond laser filamentation", Springer Series on Atomic, Optical and Plasma Physics, ISBN 978-1-4419-0687-8, Springer (2010)

2. G. Andriukaitis, T. Balčiūnas, S. Ališauskas, A. Pugžlys, A. Baltuška, T. Popmintchev, M.-C. Chen, M. M. Murnane, and H. C. Kapteyn, Opt. Lett. 36, 2755 (2011)

3. S. Henin, Y. Petit, D. Kiselev, J. Kasparian, and J.-P. Wolf, Appl. Phys. Lett. 95, 091107 (2009)

4. S. Skupin, L. Berge, Opt. Commun. 280, 173 (2007) 